\title{
Unilateral Acquired Posterior Maxillary Mandibular Syngnathia
}

\author{
Abhishek Vijay Sahu, ${ }^{1}$ Kamalika Purkayastha Roy, ${ }^{1}$ Niva Kashyap ${ }^{I}$
}

\begin{abstract}
Introduction
Congenital bony fusion of the maxilla and mandible, especially as an isolated occurrence, is a rare condition while acquired fusion being even rarer.

Case report

A rare case of a 32 year old female patient with acquired unilateral bony fusion between the zygomatic arch of maxilla and ramus of mandible is reported.

Discussion

Forty one cases of syngnathia has been reported in the international literature from 1936 to 2009, of which 39 cases are congenital and two were acquired, case 1 probably as a result of a fibrotic tubed pedicle and in case 2 from myositis ossificans. According to several case series, bony ankylosis of the mandible and maxilla has also been found to occur in $30 \%-43 \%$ of cases of NOMA.

$\underline{\text { Kevwords }}$

Syngnathia; Maxillo-mandibular
\end{abstract}

$\underline{\text { ABSTRACT }}$

$\mathrm{B}$ ony fusion of the maxilla with the mandible, also called maxillo mandibular syngnathia, is a rare clinical occurrence. It is classified into anterior or posterior, partial or complete, and unilateral or bilateral. Various synonyms for this condition include mandibulo-maxillary synostosis, congenital bony syngnathia, congenital fusion of jaws or gums, intra-oral band, intra-alveolar synechiae and zygomaticomandibular fusion. ${ }^{1,2}$

According to several case series, bony ankylosis of the mandible and maxilla has been found to occur in 30\%$43 \%$ of cases of noma. ${ }^{3}$ Two cases of acquired syngnathia have also been reported due to different causes, case 1 probably as a result of a fibrotic tubed pedicle and in case 2 from myositis ossificans. ${ }^{4}$

The aim of this report is to bring to light a very rare case of acquired maxillary mandibular syngnathia with no other intra-oral or systemic anomalies and to introspect on the ideal mode of treatment of such a case.

\section{Case report}

A 32 year old female presented with the complains of inability to open mouth since 3 years of age .The lady had no difficulty in mouth opening at birth, but at the age of 3 years she developed an abscess in the right side of the cheek on the buccal surface. There was spontaneous drainage of the pus but the trismus was progressively increasing. By 14 years of age there was complete absence of mouth opening. However, all her permanent dentition erupted at appropriate age as also her speech. She had modified her food habits and used to take only liquid and soft diet with great difficulty by pressing with her fingersand chewed it between tongue and palate. (Fig. 1)

On examination, there was reduction in lower third facial height, retrognathia of the mandible with a firm, non-tender scar in the right angle of mandible, severe limitation of mouth opening with only $6 \mathrm{~mm}$ interridge distance in the midline and left side was observed. Maxilla and mandibular dentition were maloccluded

1 - Downtown Hospital, Guwahati

Corresponding author:

Dr Abhishek Vijay Sahu

email: nehabania@yahoo.in 

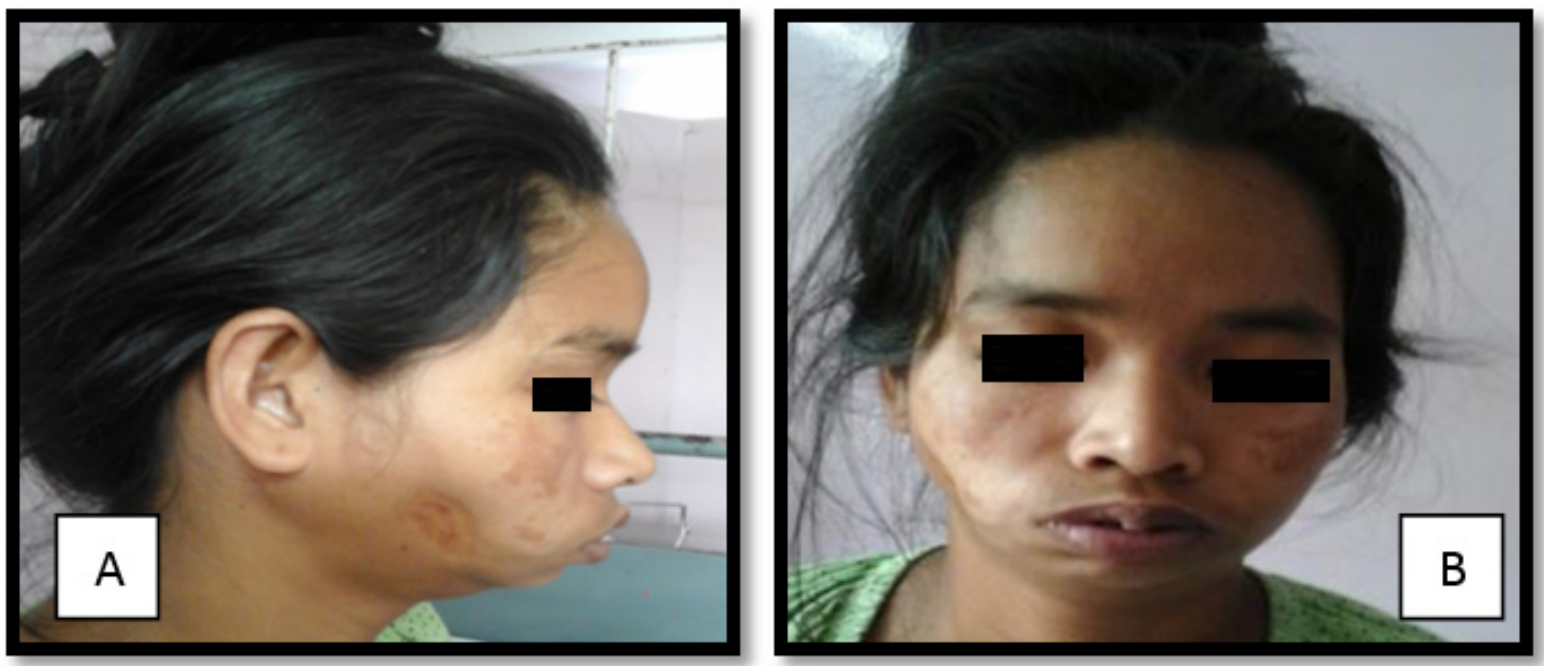

Fig.1 (A) Lateral view of face showing reduction in lower third facial height, retrognathia of the mandible. (B) Front view showing reduction of height, maloccluded maxillary and mandibular dentition.

with hypoplasia of masticator muscles of the right side. The mandible was completely immobile; however, tongue movements were normal.

The CT scan of the skull was done along with a 3D reconstruction which demonstrated bony synostosis between the zygomatic arch of maxilla and the ramus of the mandible on the right side extending from the angle of the mouth to the maxillary tuberosity in the anteriorposterior dimension and from the body of the zygoma to the mandible in the superior-inferior dimension. (Fig. 2) In addition, an exostotic mass was seen in the inner table of the right mandibular ramus. There
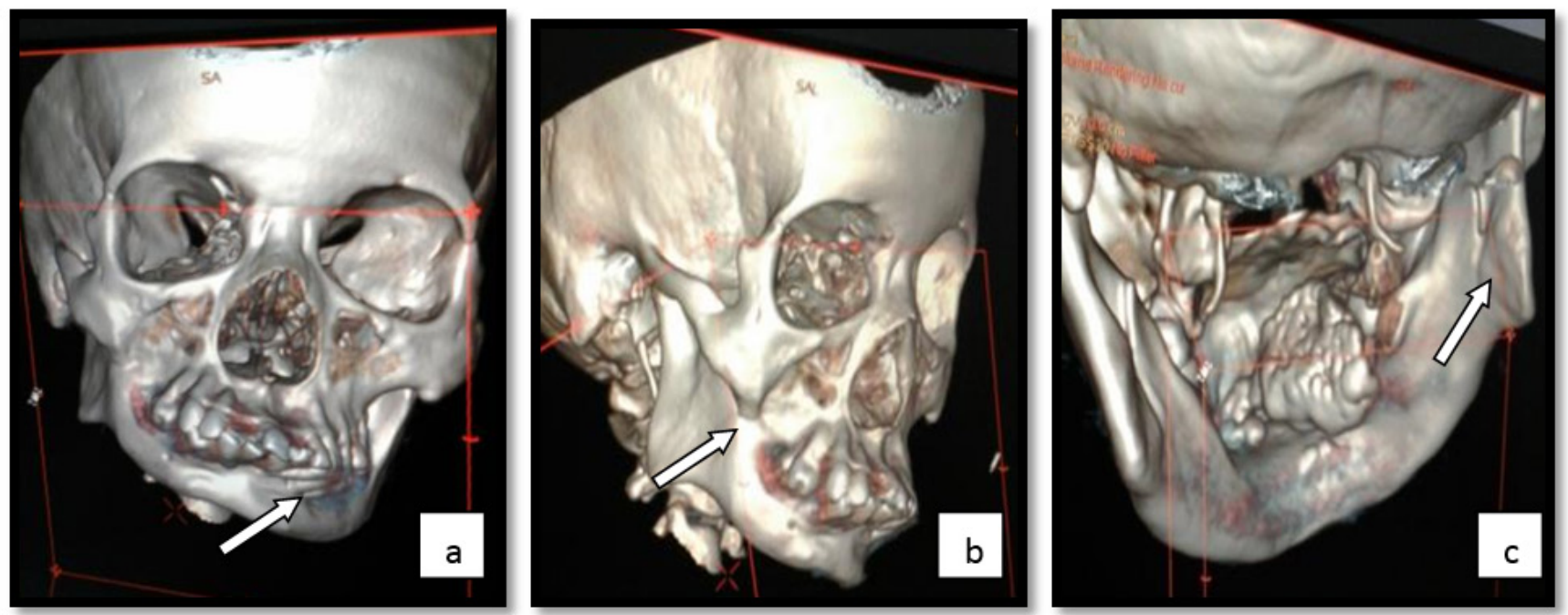

Fig.2 Reconstructed CT scan of face showing (a) Facial asymmetry, protruding upper central incisors. (b) Bony synostosis between the right zygomatic arch of maxilla and the ramus of the mandible. (c) Normal temporomandibular joint on right side is seen. 
was fatty infiltration of masseter muscles suggestive of disuse atrophy. No anatomical defects of bilateral temporomandibular joints were noted.

The patient was assessed by us in collaboration with our maxillofacial colleagues. She was planned for tracheostomy followed by release of synostosis. Since the height of the mandibular ramus would be compromised a pedicled muscle graft would be transposed. Alternately the defect could also be bridged with plate and screw. Post operatively patient would receive physiotherapy and speech therapy. But unfortunately the patient refused surgery.

\section{Discussion}

Since the first case of syngnathia was reported by Burket in $1936,{ }^{5}$ confusion exists over the incidence of this condition. Only 55 cases of congenital maxillo-mandibular fusion are reported till 2012. ${ }^{1}$ These include cases of both synechiae and syngnathia. Out of these, only 28 cases had no associated congenital or systemic defect. ${ }^{1,2,6-9}$ Forty one cases of syngnathia has been reported in the international literature from 1936 to 2009 , of which 39 cases are congenital and two were acquired, case 1 probably as a result of a fibrotic tubed pedicle and in case 2 from myositis ossificans. ${ }^{4}$ Also according to several case series, bony ankylosis of the mandible and maxilla has been found to occur in $30 \%-43 \%$ of cases of noma. ${ }^{3}$ Most reported cases were neonates with only five cases being older children and adults. ${ }^{1,2,8,10,11}$ However in this case mouth opening was decreasing progressively till age of 14 years.

Fusion of the maxilla and mandible is an extremely rare and unusual condition that can be congenital or acquired. It may be either simple soft tissue fusion (synechiae) or bony fusion (syngnathia). ${ }^{12,13}$ Soft tissue fusions of the jaws are more common and require relatively uncomplicated treatment with favorable results. Bony fusion is an extremely rare anomaly and only a handful of case reports exist in the literature. This case is an acquired syngnathia on the right side.

Fusion can be classified on basis of location into anterior or alveolar type (fusion of alveolar ridges of maxilla and mandible) and posterior or mandibulo-maxillary (fusion of ascending ramus of mandible to maxilla or zygomatic complex). Posterior fusion is less frequent than anterior bony fusion. ${ }^{14}$ The extent of fusion between the alveolar processes is variable, including complete fusion, unilateral fusion leaving a slit like opening on the opposite side of the mouth, and bilateral fusion with a small anterior slit. Review of existent literature reveals 26 cases of bilateral or complete bony syngnathia, while unilateral cases have been reported in seven instances and anterior fusion in six instances. ${ }^{1}$ A literature survey revealed just 26 cases to have been reported since 1936, of which 19 were anterior and only seven were posterior. ${ }^{15}$ Our case is an example of unilateral posterior fusion of the right side with a slit like opening on the left and an exostotic mass seen in the inner table of the right mandibular ramus.

Two classification systems have been proposed. Classification by Dawson et $\mathrm{al}^{16}$ (1997) (Table I) according to the nature of the fused tissues into either fibrous or bony. Laster et $\mathrm{al}^{17}$ (2001) modified the classification. (Table II)

In patients with syngnathia one of the major problems is the difficulty while gaining access to the airway for the purpose of intubation. Tracheostomy is the only option if other means such as blind nasal and fiber-optic bronchoscope-assisted intubation fails. ${ }^{18}$

Treatment options include surgical division of bony and soft tissue fusion and interpositions of flaps like that of temporalis muscle or buccal mucosa. Additionally, implants like silastic sheets can be inserted to prevent refusion. ${ }^{2}$ Adequate mouth opening exercises have to be performed to prevent post-operative complications of temporomandibular joint ankylosis and atrophy of muscles of mastication. However, there exists no standardized treatment protocol for this condition and surgical division has to be optimized, depending on the bony fusion. Recently, Choi et al. reported a case of using radial forearm free flap to prevent re-fusion and secure proper mouth opening after surgery in the acquired syngnathia. ${ }^{4}$ The patient in our hospital refused surgery and is started on physiotherapy with no improvement.

Although early intervention seems desirable to ensure a secure airway, reports have described repeated 
Table I : Classification of syngnathia (Dawson et al, 1997)

\begin{tabular}{|c|l|}
\hline TYPE & \multicolumn{1}{|c|}{ DESCRIPTION } \\
\hline 1 & $\begin{array}{l}\text { Simple syngnathia is bony fusion in the absence of other anomalies in the head } \\
\text { and neck }\end{array}$ \\
\hline 2 & $\begin{array}{l}\text { Complex syngnathia is bony fusion with other anomalies in the head and neck } \\
\text { a) Co-existent with aglossia } \\
\text { b) Co-existent with agenesis or hypoplasia of the proximal mandible }\end{array}$ \\
\hline
\end{tabular}

Table II : Classification of syngnathia (Laster et al, 2001)

\begin{tabular}{|c|l|}
\hline TYPE & DESCRIPTION \\
\hline $1 \mathrm{a}$ & $\begin{array}{l}\text { Simple anterior syngnathia characterized by bony fusion of the alveolar ridge } \\
\text { only and without other congenital deformity in the head and neck }\end{array}$ \\
\hline $1 \mathrm{~b}$ & $\begin{array}{l}\text { Complex anterior syngnathia characterized by bony fusion of the alveolar ridges } \\
\text { only and associated with other congenital deformity in the head and neck }\end{array}$ \\
\hline $2 \mathrm{a}$ & $\begin{array}{l}\text { Simple zygomaticomandibular syngnathia characterized by bony fusion of the } \\
\text { mandible to the zygomatic complex, causing only mandibular micrognathia }\end{array}$ \\
\hline $2 \mathrm{~b}$ & $\begin{array}{l}\text { Complex zygomaticomandibular syngnathia characterized by bony fusion of the } \\
\text { mandible to the zygomatic complex and associated with clefts or TMJ ankylosis }\end{array}$ \\
\hline
\end{tabular}

bony re-fusion after early surgery, requiring multiple surgeries. At the same time, delayed release of the maxillomandibular fusion can pose great risks such as asphyxia, aspiration pneumonia, severe malnutrition, stunted growth, poor growth of the facial skeleton, and disordered eruption of the. ${ }^{14}$

\section{Conclusion}

Our case is unique in that all rare possibilities of syngnathia in literature is present in this case i.e. it is acquired, unilateral, presenting at an age of 14 years with zygomaticomandibular (posterior) bony fusion with an exostotic mass seen in the inner table of the right mandibular ramus. Hence, more cases of this nature need to be reported to improve theoretical knowledge so that standardized management protocols can be devised and implemented.

\section{References}

1. Naikmasur VG, Sattur AP, Joshi SK, Rai A. Congenital syngnathia: Case report and review of literature. Cleft palate Craniofac J 2010; 47: 654-60

2. Kim CH, Kim MY. Congenital syngnathia: A case report. J Korean Assoc Oral Maxillofac Surg. 2012; 38: 171-6

3. Shivanand BB, Ujjwala RA, Bharat MM, Gundareddy NS, Shruti G. Bony fusion of the maxilla and mandible as a sequelae of noma: A rare case report. Imaging Science in Dentistry 2015; 45: $193-8$

4. Choi JY, Min CG, Myoung H, Hwang SJ, Kim MJ, Lee JH. 
Acquired syngnathia. Br J Oral Maxillofac Surg. 2004; 42: 44850

5. Burket LW. Congenital bony temporo-mandibular ankylosis and facial hemiatrophy. JAMA 1936; 106: 1719-22

6. Heqab A, Madawy AE, Shawkat WM. Congenital maxillo-mandibular fusion: A report of three cases. Int J Oral Maxillofac Surg. 2012; 41: 1248-52

7. El-Hakim IE, Al-Sebaei MO, Abuzennada S, AlYamani AO. Congenital fusion of the maxilla and mandible (congenital bony syngnathia). Int J Oral Maxillofac Surg. 2010; 39: 933-6

8. Halli R, Kharkar V, Kini Y, Rudaqi BM. Congenital unilateral maxillo- mandibulo- zygomatic fusion (syngnathia): A case report in an 8-year-old boy. Int J Oral Maxillofac Surg. 2010; 39: $500-2$

9. Subramanian B, Agrawal K, Panda K. Congenital fusion of the jaws: A management protocol. Int J Oral Maxillofac Surg. 2010; 39: 925-9

10. Koeda S, Suzuki T, Nei H, Inahara H, Takata Y, Goto S. A case of congenital unilateral maxillo-mandibular bony fusion in an 8-year-old girl. Asian J Oral Maxillofac Surg. 2010; 22: 220-4

11. Turksen Z, Ozakpinar HR, Tellioglu AT. A case of syngnathia, cleft palate and hypospadias: An isolated case or syndromic syngnathism? J Craniomaxillofac Surg. 2012; 40: 8-10
12. Yazdi I, Fakhraee AH. Congenital fusion maxilla and mandible (bony syngnathia): A case report. Br J Plast Surg. 2000; 3:3

13. Kenneth H, Myall D, Myall RW. Congenital bony syngnathia: A proposed classification. Cleft Palate Craniofac J 1997; 34 241-6

14. Vahidi K, Joe BN, Glenn OA, Barkovich AJ, Filly R, Ball R. Prenatal imaging of congenital maxillo-mandibular fusion: Case report and review of the literature. J Ultrasound Med. 2007; 26 : 381-9

15. Daniels JSM. Congenital maxillomandibular fusion, a case report and review of the literature. J Craniomaxillofac Surg. 2004; 32: 135-9

16. Dawson KH, Gruss JS, Myall RW. Congenital bony syngnathia: a proposed classification. Cleft Palate Craniofac J 1997: 34: 141-6

17. Laster Z, Temkin D, Zarfin Y, Kushnir A. Complete bony fusion of the mandible to the zygomatic complex and maxillary tuberosity: case report and review. Int J Oral Maxillofac Surg. 2001: 30: 75-9

18. Alfery DD, Ward CF, Harwood IR, Mannino FL. Airway management of neonate with congenital fusion of the jaws. Anaesthesiology. 1979; 51: 340-2. 